\title{
Cranial Nerve VI Palsy as Presenting Sign of Previously Undiagnosed Metastatic Prostate Adenocarcinoma to the Clivus
}

\author{
Jennifer E. Douglas ${ }^{a} \quad J o h n$ Y.K. Lee ${ }^{b} \quad$ Karthik Rajasekaran ${ }^{a}$ \\ ${ }^{a}$ Department of Otorhinolaryngology - Head \& Neck Surgery, University of Pennsylvania \\ Health System, Philadelphia, PA, USA; ${ }^{b}$ Department of Neurosurgery, University of \\ Pennsylvania Health System, Philadelphia, PA, USA
}

\section{Established Facts}

- Prostate adenocarcinoma is the most common malignancy in males in the United States.

- Metastatic prostate adenocarcinoma affects the bone in approximately $86 \%$ of the cases.

\section{Novel Insights}

- Metastatic prostate adenocarcinoma can involve the skull base in unique circumstances.

- Cranial neuropathies are a chief presenting sign of metastatic disease involving the skull base.

\section{Keywords}

Skull base $\cdot$ Sellar mass · Clival metastasis · Cranial neuropathy · Prostate adenocarcinoma

\begin{abstract}
Prostate adenocarcinoma is the most common malignancy in males in the United States and is typically highly treatable. Herein we present a case report of a male with a history of prostate adenocarcinoma previously managed with definitive radiation therapy who presented with sudden onset diplopia and examination consistent with an abducens palsy. He was ultimately found to have prostate adenocarcinoma metastatic to the clivus causing cranial neuropathy, and was referred for systemic chemotherapy and palliative Cyberknife stereotactic radiosurgery. While relatively rare, metastatic disease should be included in the differential diagnosis of atypical skull base lesions.

(C) 2020 The Author(s)

Published by S. Karger AG, Basel
\end{abstract}

This research was presented at the North American Skull Base Society Meeting in San Antonio, TX, USA, on February 7, 2020. 


\section{What Is It about?}

Prostate adenocarcinoma is the most common malignancy affecting males in the United States, with metastatic disease affecting the bone in the majority of cases. In unique circumstances, metastatic prostate adenocarcinoma can involve the skull base. Herein we present a unique case report of a male who presented with an abducens palsy as the first presenting sign of what was found to be recurrent, metastatic prostate adenocarcinoma involving the clivus.

\section{Introduction}

Prostate adenocarcinoma is the most common cancer in males in the United States with an annual incidence of 110 per 100,000 males annually [1]. Typically, highly curable, prostate adenocarcinoma is the second leading cause of cancer-related death due to metastatic disease to the bone in $86 \%$ of the cases [2]. There has, however, been only 3 reported cases of metastatic prostate adenocarcinoma to the clivus [3-5]. Herein we present the case of a male who presented with a cranial nerve VI palsy with imaging showing an infiltrative skull base mass, who was ultimately diagnosed with prostate adenocarcinoma metastatic to the clivus.

\section{Case Report}

An 81-year-old male with a history of prostate adenocarcinoma status-post radiation 3 years prior to presentation presented to the emergency department for evaluation of suddenonset diplopia. Of note, the patient was on enzalutamide for rising prostate-specific antigen in the absence of known active disease. His examination showed a partial right abducens palsy on extreme lateral gaze, and he had no other focal neurological deficits. A non-contrast computed tomography scan of the sinuses was performed, which showed a $2.5 \times 3.5 \times 3.9 \mathrm{~cm}$ midline skull base mass involving the sella and suprasellar regions (Fig. 1a), and he was subsequently admitted to the hospital for further workup. He was unable to undergo a magnetic resonance imaging (MRI) at that time as it was thought that his stainless steel prostate seeds were a contraindication. Therefore, based on the CT scan, differential included pituitary adenoma, meningioma, hematologic malignancy, chondrosarcoma, chondroma, and metastatic disease. Endocrinology was consulted given concern for secreting pituitary adenoma, with no evidence of endocrine dysfunction.

Due to inability to perform further imaging, decision was made to take the patient to the operating room and obtain tissue biopsy for definitive diagnosis. An anterior skull base approach was performed, the sella was entered, and biopsies were taken. Intraoperative frozen section showed atypical epithelioid neoplasm, with final pathology confirming a diagnosis of metastatic prostatic adenocarcinoma. He was subsequently cleared to undergo MRI scan, which demonstrated an infiltrative mass of the clivus extending to the petrous apices, cavernous sinus, petrous and cavernous internal carotid arteries, prepontine cisterns, and abutting the pituitary gland (Fig. 1b).

The patient was referred to medical oncology and radiation oncology for systemic therapy and palliative radiation therapy. He was initiated on systemic therapy at his local hospital including leuprolide, denosumab, and abiraterone, and received a total dose of 3,000 cGy in 5 fractions of Cyberknife stereotactic radiosurgery. At his 3-month post-treatment visit, he 


\section{Biomedicine \\ Hub}

\begin{tabular}{|c|c|}
\hline \multicolumn{2}{|l|}{ Biomed Hub 2020;5:510303 } \\
\hline DOI: $10.1159 / 000510303$ & $\begin{array}{l}\text { (c) } 2020 \text { The Author(s). Published by S. Karger AG, Basel } \\
\text { www.karger.com/bmh }\end{array}$ \\
\hline
\end{tabular}

Douglas et al.: Cranial Nerve Palsy as Sign of Clival Metastatic Disease
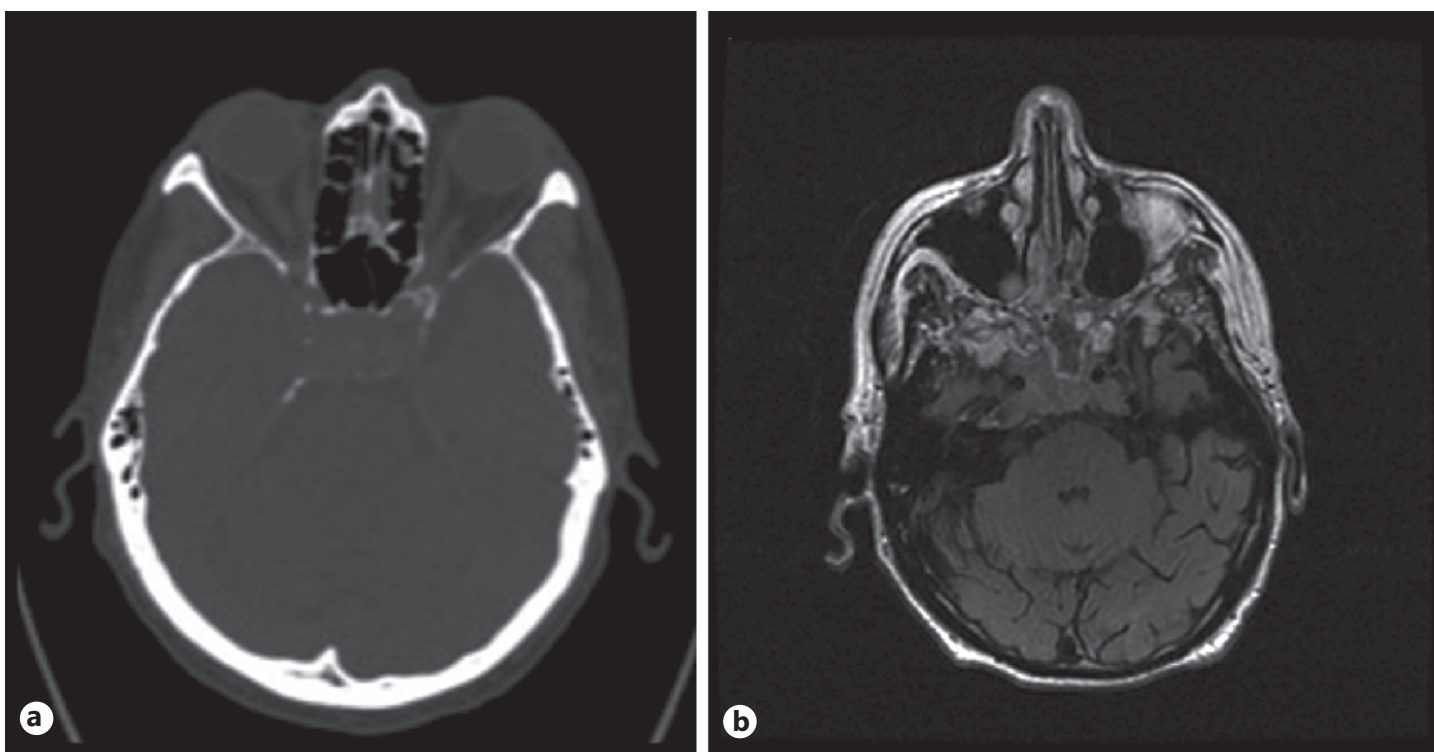

Fig. 1. a Pre-treatment non contrast-enhanced computed tomography scan at the level of the pituitary gland showing a $2.5 \times 3.5 \times 3.9 \mathrm{~cm}$ midline skull base mass involving the clivus, sella, and suprasellar regions. b Pre-treatment T2-weighted fluid-attenuated inversion recovery MRI of the head demonstrating diffuse metastatic infiltration and destruction of the clivus.

Fig. 2. Three-month post-treatment T2-weighted fluid-attenuated inversion recovery MRI of the head with and without IV contrast demonstrating invasive, destructive metastatic disease of the clivus without significant change.

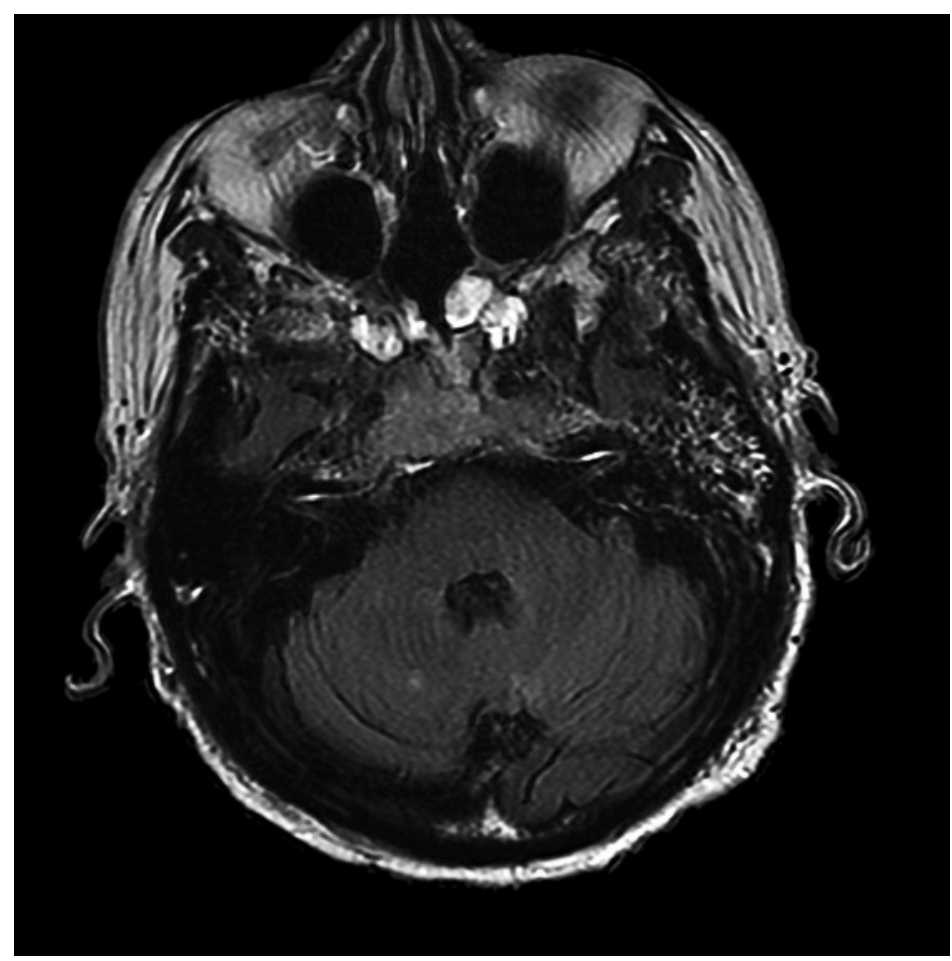

reported intermittent blurry vision and diplopia, with examination notable for persistent partial right abducens palsy. His Eastern Cooperative Oncology Group (ECOG) performance status was grade one, with restriction in strenuous activity but otherwise intact. MRI brain with and without IV contrast was notable for invasive, destructive metastatic disease of the clivus, 
which was concerning for possible dural-based progression (Fig. 2). However, given his clinical stability, plan was made for follow-up in 3 months. He continues on systemic chemotherapy.

\section{Conclusion}

Prostate adenocarcinoma is the leading cause of cancer in males, and metastatic disease can involve the skull base in unique circumstances. Few case reports exist of prostate adenocarcinoma metastatic to the clivus. Herein we add to this literature by presenting a case of a patient with isolated cranial nerve VI palsy as the presenting sign of previously undiagnosed metastatic prostate adenocarcinoma involving the clivus. The skull base surgeon should maintain a high clinical suspicion for metastatic disease as a cause of atypical sellar and suprasellar lesions.

\section{Statement of Ethics}

This case report was exempt from review by the Institutional Review Board at the University of Pennsylvania, and the patient gave written informed consent for publication of his case.

\section{Conflict of Interest Statement}

The authors have no conflicts of interest to disclose.

\section{Funding Sources}

The authors have no relevant funding sources.

\section{References}

1 Howlader N, Noon AM, Krapcho M, Miller D, Brest A, Yu M, et al., editors. SEER Cancer Statistics Review, 1975 2016, National Cancer Institute, Bethesda, MD. https://seer.cancer.gov/csr/1975_2016/, based on November 2018 SEER data submission, posted to the SEER web site, April 2019.

2 Gandaglia G, Abdollah F, Schiffmann J, Trudeau V, Shariat SF, Kim SP, et al. Distribution of metastatic sites in patients with prostate cancer: A population-based analysis. Prostate. 2014 Feb;74(2):210-6.

3 Özbek Z, Özkara E, Arik D, Ant MA. Calvarial-orbital Metastasis of Prostate Carcinoma which was Diagnosed with Sixth Cranial Nerve Palsy. Asian J Neurosurg. 2017 Oct-Dec;12(4):769-71.

4 O’Boyle JE, Gardner TA, Oliva A, Enzenauer RW. Sixth nerve palsy as the initial presenting sign of metastatic prostate cancer. A case report and review of the literature. J Clin Neuroophthalmol. 1992 Sep;12(3):149-53.

5 Malloy KA. Prostate cancer metastasis to clivus causing cranial nerve VI palsy. Optometry. 2007 Feb;78(2): 55-62. 